\title{
Selective attention is a primary determinant of the relationship between working memory and general learning ability in outbred mice
}

\author{
Stefan Kolata, Kenneth Light, Henya C. Grossman, Gregory Hale, \\ and Louis D. Matzel ${ }^{1}$ \\ Department of Psychology, Rutgers University, Piscataway, New Jersey 08854, USA
}

\begin{abstract}
A single factor (i.e., general intelligence) can account for much of an individuals' performance across a wide variety of cognitive tests. However, despite this factor's robustness, the underlying process is still a matter of debate. To address this question, we developed a novel battery of learning tasks to assess the general learning abilities (GLAs) of mice. Using this battery, we previously reported a strong relationship between GLA and a task designed to tax working memory capacity (i.e., resistance to competing demands). Here we further explored this relationship by investigating which aspects of working memory (storage or processing) best predict GLAs in mice. We found that a component of working memory, selective attention, correlated with GLA comparably to working memory capacity. However, this relationship was not found for two other components of working memory, short-term memory capacity and duration. These results provide further evidence that variations in aspects of working memory and executive functions covary with general cognitive abilities.
\end{abstract}

Most animal studies of learning and memory focus on a single domain of learning, such as spatial ability. While such studies are of great virtue in elucidating the mechanisms of those specific systems, they tend to ignore the aspects of learning that are shared by all learning tasks. To address this, we have previously developed a battery of tasks that are sensitive to a general learning factor in mice. Specifically, we found that as much as $38 \%$ of the variance in the performances of mice across ostensibly unrelated learning tasks, which impinge on different domains of learning, can be explained by a single factor (Matzel et al. 2003). In other words, while any given learning task draws on domain specific abilities, there is some common factor that influences performance in all tasks. In many ways, this factor in mice is analogous to general "intelligence" in humans (Blinkhorn 2003). Indeed, studies have linked learning abilities in humans to general intelligence (Ackerman 1987, 2005). However, similar to general intelligence, the underlying basis of general learning abilities remains elusive.

While general intelligence in humans may be more complex then are general learning abilities in mice, it seems reasonable that they may share some of the same underlying mechanisms and/or reflect a similar psychological process. One theory of general intelligence that has gained particular popularity holds that individual differences in working memory capacity underlie differences in general intelligence. Working memory is a limited capacity system that maintains and processes information relevant to an ongoing task, often under conditions of competing demands (Baddeley 1986, 2003). It is distinct from the concept of short-term memory in that it involves both maintenance as well as processing of information over a short period of time (Baddeley 1986; Jarrold and Towse 2006). Thus it is thought to be engaged by virtually all tests of cognitive abilities (Mackintosh 1998). It is this aspect of working memory that makes it so appealing to theorists attempting to explain the mechanisms of

'Corresponding author.

E-mail matzel@rci.rutgers.edu; fax (732) 445-2263.

Article is online at http://www.learnmem.org/cgi/doi/10.1101/lm.408507. general intelligence. Indeed, empirical studies have indicated that working memory capacity covaries with general intelligence (Conway and Engle 1995; Engle et al. 1999; Süß et al. 2002; Colom et al. 2004). In addition, recent imaging studies have shown that a wide range of cognitive tests engage areas of the frontal cortex (particularly the dorsolateral prefrontal cortex), which are thought to be the neural substrates for aspects of working memory (Gray et al. 2004; Haier et al. 2004). In fact, activity in the dorsolateral prefrontal cortex during intelligence tests is predictive of performance on those tests (Conway et al. 2003).

We previously investigated the relationship between general learning abilities in mice and working memory (Kolata et al. 2005). The tasks that comprise the learning battery (e.g., Lashley III maze, passive avoidance, spatial water maze, odor discrimination, fear conditioning) were explicitly chosen so that each one places unique sensory, motor, motivational, and information processing demands on the animals. Briefly, performance in the Lashley III maze depends on animals' use of fixed motor patterns (egocentric navigation) motivated by a search for food. Passive avoidance is an operant conditioning paradigm in which the animals must learn to be passive in order to avoid aversive light and noise stimulation. The spatial water maze encourages the animals to integrate spatial information to efficiently escape from a pool of water. Odor discrimination is a task in which animals must discriminate and use a target odor to guide their search for food. Finally, fear conditioning (assessed by behavioral freezing) is a conditioning test in which the animals learn to associate a tone with a shock.

We reported a positive correlation between the aggregate performance of individual outbred mice in the learning battery described above and their subsequent ability to accommodate competing demands on their spatial working memory capacity (Kolata et al. 2005). Specifically, we observed that when mice were required to perform in two radial arm mazes concurrently (a manipulation intended to place demands on working memory capacity), their performance in the target maze positively correlated with their general learning abilities. These results are suggestive of a relationship between working memory capacity and 
Table 1. Tasks used to isolate different aspects of working memory

\begin{tabular}{llcl}
\hline Task & $\begin{array}{c}\text { Selective } \\
\text { attention }\end{array}$ & $\begin{array}{c}\text { Short-term } \\
\text { memory } \\
\text { duration }\end{array}$ & $\begin{array}{c}\text { Short-term } \\
\text { memory } \\
\text { capacity }\end{array}$ \\
\hline $\begin{array}{l}\text { Complex discrimination } \\
\begin{array}{l}\text { Delayed reinforced alternation } \\
\text { Nonspatial radial arm maze }\end{array}\end{array}$ & $\begin{array}{llc}\text { High } \\
\text { Low }\end{array}$ & Low & Low \\
\hline
\end{tabular}

general learning abilities in mice. However, working memory is not a unitary process; it is thought to encompass both short-term storage of information as well as other systems involved in active processing and integrating of information (Baddeley 1986, 2003; Jarrod and Towse 2006). Our previous experiment could not discern the relative contributions of these various aspects of working memory to general learning abilities. Thus subsequent experiments are needed to further understand this relationship.

The present study was designed to independently assess the relative contributions of the processing and storage aspects of working memory to general learning ability. Specifically, we assessed animals' general learning abilities and then subsequently measured two aspects of storage (short-term memory duration and span) as well as one measure of processing (selective attention). Short-term memory duration is thought of as the length of time that information can be maintained in active memory, whereas span is the number of items that can be held in memory. Selective attention is the ability to attend to task-relevant cues while ignoring distracters. While no task can be process-pure, that is to say, tax only one cognitive system in isolation, each task was designed to draw most heavily on one specific component of working memory (Table 1). It was hypothesized that if the previously reported relationship between general learning abilities and working memory was due more to one of these working memory components, we would find differential patterns of correlation between the manipulations and general learning ability.

\section{Results}

In all of the learning tasks, animals' performance was measured during acquisition, where there are considerable individual differences in performance. In the tasks in which there was only one testing trial (fear conditioning and passive avoidance), we used training parameters that we previously showed to result in subasymptotic responding during testing (Matzel et al. 2003). The correlations between each of the learning tasks revealed a positive manifold, i.e., all correlations were consistently positive, suggesting a common source of variance (Table $2 \mathrm{~A}$ ). A subsequent principal component factor analysis revealed that 43\% (Eigen value $=2.63)($ Table $2 \mathrm{~B})$ of the variations in the animals' performances between the tasks could be explained by a single factor ("general learning" factor). While a sample size of 27 is generally considered small for the application of factor analytic techniques, the results were consistent with what has previously been shown using substantially larger samples (Matzel et al. 2003).

In order to quantify individual animals' general learning abilities, we calculated factor scores for each animal. A factor score is analogous to the average z-score for each animal in each task, weighted by the degree to which each task contributes to the primary factor (the general learning factor) in the factor analysis. Thus, a factor score of zero would indicate an "average" learner, while a low score would be indicative of a fast learner; conversely, a high score would designate a poor learner. Previously we have used a simple average rank to classify animals relative to each other within a sample; however, since each task in the battery contributes to varying degrees to the primary factor, factor scores better capture the "general learning" abilities of individual animals. In the present sample, the factor score ranged from -2.24 to 1.89 (factor scores always have a mean of zero and a standard deviation of one).

Analogous to the wide range of general learning differences observed between the animals in the learning battery, our measure of short-term memory duration in the reinforced alternation task revealed a broad gamut of abilities. For instance, while some animals could withstand only a 60 -sec delay between choices, others could tolerate nearly three and a half times as long an interval. The distribution of short-term memory durations was normally distributed around a mean of $115 \mathrm{sec}$ (Shapiro-Wilk's test (0.917), $P<0.05)$. However, there was no significant correlation between this ability and the animals' general learning ability score $(r=0.15, P=\mathrm{ns})$ (Fig. 1$)$. In fact, this relationship, although nonsignificant, was in the opposite direction one would have predicted if general learning abilities were in fact dependent on short-term memory duration, where those animals that showed the fastest learning tended to have the shortest short-term memory durations.

Our other measure of the storage component of working memory was obtained in the nonspatial working memory task. In this task, the animals had to remember the visual cues (out of a total of six) at which they had already found food during that session. Thus, their asymptotic performance was thought to measure their visual short-term memory span. In this task we found overall poorer learning then was expected a priori. In the initial trial (with zero knowledge of the task's contingencies), the animals made on average 13.1 errors. However, the animals never reached perfect performance at asymptote; on average, at that

Table 2A. A correlation matrix of all of the tasks in the battery in experiment 2 shows that they correlate consistently with the selective attention manipulations but not the storage components of working memory

\begin{tabular}{|c|c|c|c|c|c|c|c|c|c|}
\hline & LM & PA & WM & FC & VD & $O D$ & STM: Cap & STM: Dur & SELATTEN \\
\hline LM & & $0.72^{* *}$ & $0.43^{*}$ & 0.13 & 0.28 & 0.35 & 0.28 & 0.13 & $0.64^{* \star}$ \\
\hline PA & $0.72^{\star *}$ & & 0.36 & 0.07 & 0.29 & $0.41^{*}$ & 0.28 & 0.16 & $0.56^{* *}$ \\
\hline WM & $0.43^{*}$ & 0.36 & & 0.14 & $0.44^{*}$ & 0.28 & 0.20 & 0.06 & 0.24 \\
\hline FC & 0.13 & 0.07 & 0.14 & & 0.13 & 0.26 & -0.06 & -0.10 & 0.14 \\
\hline VD & 0.28 & 0.29 & $0.44^{*}$ & 0.13 & & 0.06 & 0.35 & 0.21 & 0.23 \\
\hline OD & 0.35 & $0.41^{*}$ & $0.28^{*}$ & 0.26 & 0.06 & & 0.29 & 0.07 & $0.61^{* *}$ \\
\hline STM: Cap & 0.28 & 0.28 & 0.20 & -0.06 & 0.35 & 0.29 & & 0.08 & 0.27 \\
\hline STM: Dur & 0.13 & 0.16 & 0.06 & -0.10 & 0.21 & 0.07 & 0.08 & & -0.16 \\
\hline SELATTEN & $0.64^{\star *}$ & $0.56^{* *}$ & 0.24 & 0.14 & 0.23 & $0.61^{* *}$ & 0.27 & -0.16 & \\
\hline
\end{tabular}

LM indicates Lashely maze; PA, passive avoidance; WM, spatial water maze; FC, fear conditioning; VD, visual discrimination; OD, odor discrimination; STM: Cap, performance in task measuring short-term memory capacity; STM: Dur, performance in task measuring short-term memory duration; SELATTEN, performance in task assessing selective attention. ${ }^{*} P<0.05,{ }^{*} P<0.01$. 
Table 2B. An unrotated factor analysis extracted from this correlation matrix reveals that all of the learning tasks load positively on a primary factor (general learning ability) which accounts for $44 \%$ of the variance

\begin{tabular}{lcr}
\hline & Factor 1 & Factor 2 \\
\hline Lashley Maze & 0.83 & -0.01 \\
Passive Avoidance & 0.82 & 0.02 \\
Water Maze & 0.73 & -0.28 \\
Fear Conditioning & 0.34 & 0.49 \\
Visual Discrimination & 0.60 & -0.54 \\
Odor Discrimination & 0.52 & 0.68 \\
\hline Eigen Value & 2.63 & 1.06 \\
Proportion of Total Variance & 0.44 & 0.18 \\
\hline
\end{tabular}

point they were still making seven errors per trial (Fig. 2A). Nevertheless, all animals did improve over trials, reaching a consistent level of performance at which point a wide range of individual differences were observed. When we compared their performance at this state with their general learning abilities, we found a significant correlation in the direction one would expect if better learners tended to make fewer errors at asymptotic levels of performance ( $r=0.38, P<0.05)$ (Fig. 2B).

While the measure of short-term memory duration failed to show a relationship with general learning ability, and the measure of short-term memory span showed only a moderate correlation with general learning abilities, this was not the case for our measure of the processing component of working memory (selective attention). In this task, the animals performed a complex discrimination in which they had to attend to one dimension (e.g., odor or visual) and ignore salient distracting cues. They performed both a visual discrimination task with odor distracters and an odor discrimination task with visual distracters. Each animal's selective attention score was an average of the number of errors they made on trials 4-6 in both the visual discrimination with odor distracters condition and the odor discrimination with visual distracters condition. We found a significant relationship between the animals' general learning abilities and their capacity to ignore salient distracters while performing a discrimination task $(r=0.50, P<0.01)$ (Fig. 3$)$. It is important to note that in both the visual discrimination and the odor discrimination tasks, there was no overall relationship between the animals' performance without the distracters (at asymptote) and their learning abilities $(r=-0.05, P<\mathrm{ns}$ and $r=-0.14, P<\mathrm{ns})$. This indicates that the animals' ability to ignore task-irrelevant distracters, not the task demands per se, accounted for the correlation between selective attention and general learning abilities.

In order to better understand the relationship between general learning abilities and both the storage components of working memory (as assessed in the delayed reinforced alternation and the nonspatial radial arm maze) as well as the processing component (as assessed in the selective attention task), a principal component factor analysis containing the animals' performance during those tasks and their performance in the learning battery was performed. It was found that the duration of shortterm memory did not load consistently with the learning tasks on the primary factor. Nevertheless, slightly higher loadings for the measure of short-term memory span on the first factor were observed. These patterns of results were contrasted with the strong loading for the measure of selective attention on the primary factor. No obvious pattern of loadings was detectable in the second factor extracted from this analysis (Table 2C).

\section{Discussion}

We previously reported that working memory capacity covaries with the general learning abilities of genetically diverse outbred mice (Kolata et al. 2005). This finding is consistent with the hypothesis that working memory contributes broadly to a wide range of cognitive processes. However, most contemporary models suggest that the working memory system involves at least two distinct aspects-a short-term storage component and a processing component (Baddeley 1986, 2003). In our previous work, we did not determine the relative contributions of these two aspects of working memory to general learning ability. The present study was therefore designed with the intention of separating these specific components of working memory and to determine their differential relationships to general learning abilities.

Short-term memory differs from working memory in that short-term memory is only one component of the broader process that constitutes the working memory system. Working memory involves not just storage but also processing and integration of information in a goal-dependant fashion (Jarrold and Towse 2006). The results from the present study are suggestive that the storage aspect of working memory plays at best a minimal role in determining individual differences in learning.

While there was no relationship found between short-term memory duration and learning, a moderate relationship between short-term memory span and general learning abilities was observed. This result suggests the possibility that our previously reported correlation between working memory capacity and general learning abilities may be due in part to the short-term memory component of working memory. However, this may not be the most likely scenario. It is very difficult to isolate just shortterm memory independent of processing demands in nonverbal animals such as mice. While we attempted to minimize any processing component of this task, it is impossible to do so entirely. Thus given the relatively small correlation between this task and general learning abilities, it is difficult to conclude that shortterm memory is a critical component of general learning abilities, although this possibility cannot be ruled out.

A critical relationship was observed between our measure of selective attention and general learning ability. A contribution of selective attention to general learning ability is parsimonious with contemporary views of working memory and intelligence. That which determines general learning abilities must not be unique to one type of learning or one sensory modality. The existence of distinct circuits in the brain that are specialized for processing certain types of information is seemingly at some

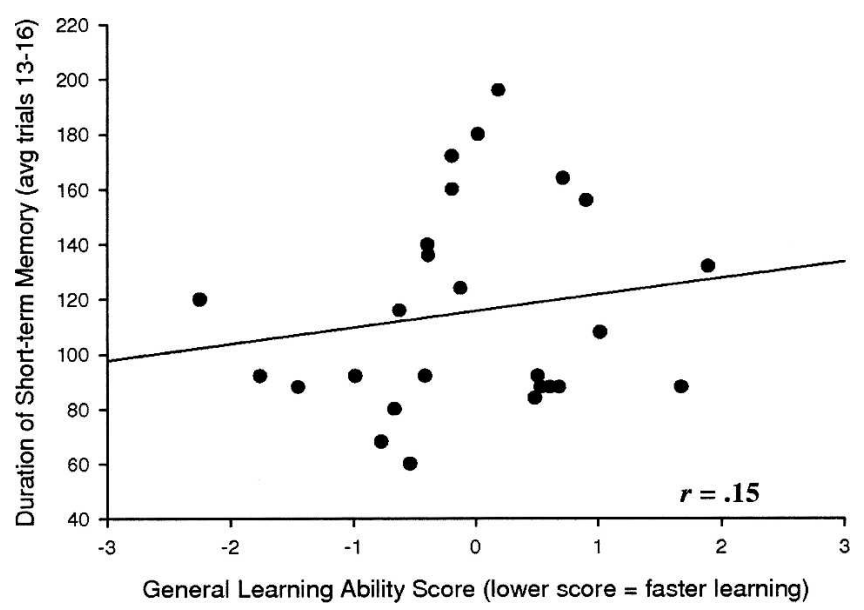

Figure 1. Short-term memory duration and general learning ability. Correlation between the animals' average memory span in the reinforced alternation and their performance in the learning battery. If there was a relationship between the learning battery performance and short-term memory delay, it would be expected that the longest delays would cluster around the lowest ranks (best learners). This is not what was seen.

\section{Learning \& Memory}

www.learnmem.org 
A

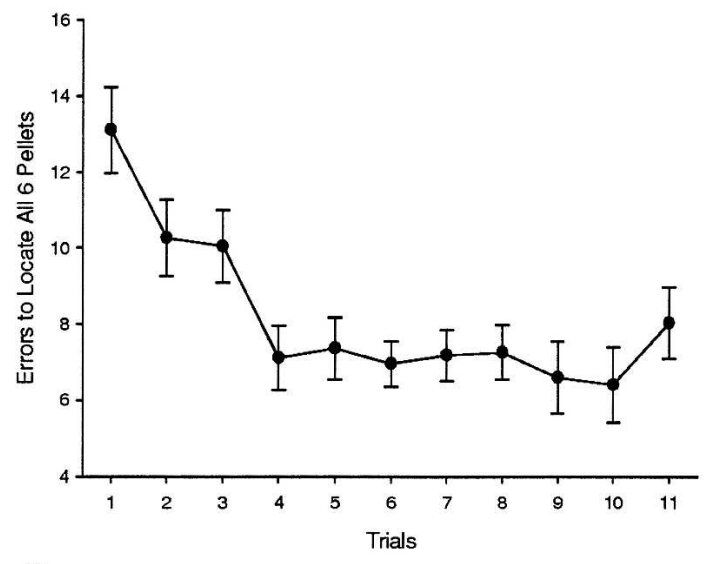

B

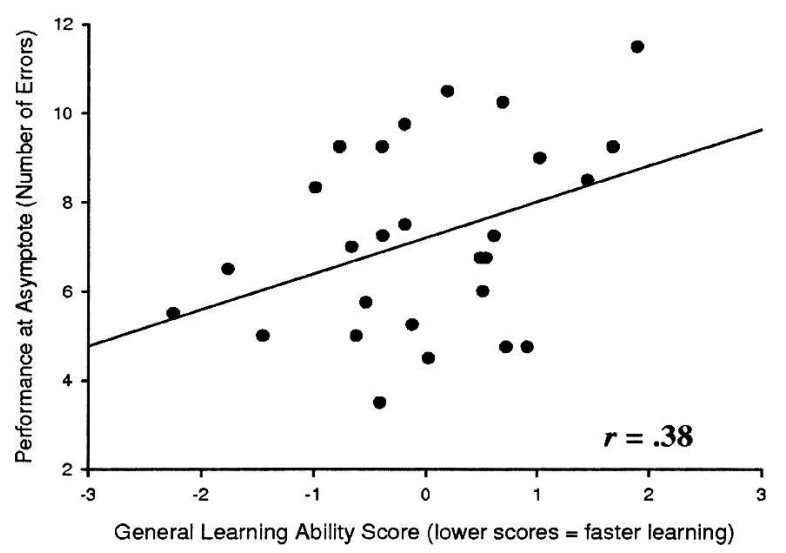

Figure 2. Short-term memory capacity and general learning ability. ( $A$ ) Acquisition data for 27 mice in the nonspatial radial arm maze. (B) Correlation between the animals' performance at asymptote (average trials $=5-8$ ) in the nonspatial radial arm maze task and their performance in the learning battery.

level inconsistent with the very idea of a general learning factor. However, animals have limited resources and cannot attend equally to all the stimuli in their environment at one time. Since they cannot know a priori (i.e., on the initial training trial) which type of stimuli are relevant to the task at hand, all learning is dependant to some degree on the ability to decide the relative predictive nature of stimuli and to only attend to those that reliably predict the relevant outcome. In other words, all learning may be to some degree dependant on selective attention (Dayan et al. 2000).

In our selective attention task, the animals had prior experience with a visual cue and an odor cue, both of which predicted reward in distinct environmental contexts. When the cues were combined, only one modality reliably predicted food in the test context (e.g., in the visual discrimination with odor distracters task only the visual cues predicted food). In order to perform this task efficiently, the animals had to selectively attend to those cues that were of greater predictive value in that environment. Given both the conceptual basis for a relationship between selective attention and general learning abilities as well as the strong positive correlation between the variables observed here, it is reasonable to suppose that there is a causal relationship between selective attention and general learning abilities.

The relationship between general learning abilities and selective attention could be interpreted as indicating that our gen- eral "learning" battery is in reality only isolating attentional abilities and that if we had chosen a different set of tasks (i.e., ones not dependant on selective attention), we may have not observed a general "learning" factor. This may be the case, but only to the extent that any task (or set of tasks) could be devoid of attentional demands. However, it is important to note that the tasks in the general learning battery were not chosen a priori because of the demands they placed on attention. Instead, they were selected because they represent a disparate set of commonly used learning tasks that each places unique sensory, motor, motivational, and information processing demands on the animals (Matzel et al. 2003). Therefore, it seems more likely that the results presented here suggest that selective attention may be a primary determinant of overall learning abilities because most, if not all, learning tasks require (to varying degrees) selective attention.

It could be argued that instead of isolating the relationship between working memory and general learning abilities, all the tasks share in common "complexity," and therefore, any cognitive task with sufficient difficulty would show a positive correlation with our "general learning" factor. However, it is difficult to see how the delayed reinforced alternation task is any less complex or difficult then any of the learning tasks such as fear conditioning. Therefore, if difficulty was the main determinant of which tasks loaded in the primary factor, one would have expected delayed reinforced alternation to have loaded in the same direction as the learning tasks.

Overall, the present work sets the ground work for exploring the neurobiological basis of general learning ability in mice, with an eye toward a better understanding of general cognitive ability in humans. Understanding the key factors that underlie all learning may help in designing better treatments for learning disabilities. Our results also provide further support for the existence of common mechanisms that affect all learning systems across species (Locurto and Scalon 1998; Conway et al. 2002; Galsworthy et al. 2002; Locurto et al. 2003; Matzel et al. 2003).

\section{Materials and Methods}

\section{Subjects}

The 27 CD-1 mice (Harlan Sprague Dawley) used in this experiment were between 25 and $35 \mathrm{~g}$ upon arrival. They were housed individually in clear shoebox cages in a temperature and humidity-controlled colony room and were maintained on a 12-h light/

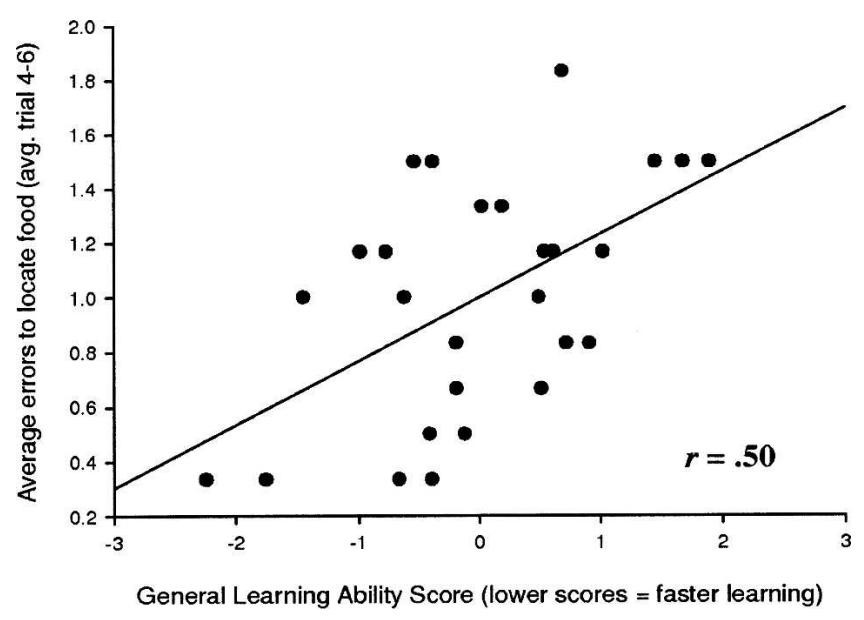

Figure 3. Selective attention and general learning ability. Correlation between the animals' performance during trials $4-6$ of visual discrimination with distracter odor cues present and general learning abilities. 
Table 2C. An unrotated factor analysis extracted from this correlation matrix reveals that both selective attention manipulation load positively in the same factor as the tasks in the battery. To a lesser degree, the measurement of short-term memory capacity, but not short-term memory duration, also loads in this factor

\begin{tabular}{lcr}
\hline & Factor $\mathbf{1}$ & Factor 2 \\
\hline Lashley Maze & 0.82 & -0.02 \\
Passive Avoidance & 0.80 & 0.02 \\
Water Maze & 0.60 & 0.23 \\
Fear Conditioning & 0.13 & -0.40 \\
Visual Discrimination & 0.52 & 0.52 \\
Odor Discrimination & 0.65 & -0.36 \\
Short-term Memory Capacity & 0.50 & 0.28 \\
Short-term Memory Duration & 0.14 & 0.68 \\
Selective Attention & 0.78 & -0.40 \\
\hline Eigen Value & 3.30 & 1.33 \\
Proportion of Total Variance & 0.37 & 0.15 \\
\hline
\end{tabular}

12-h dark cycle. In order to minimize any effect of individual differences in stress reactivity to handling, prior to the start of the experiment all of the animals were handled for $90 \mathrm{sec}$ a day for 5 $\mathrm{d}$ a week over a period of $2 \mathrm{wk}$. During the final task, reinforced alternation, one of the subjects became sick and was unable to complete the task.

\section{Behavioral training and testing}

\section{Assessing general learning abilities}

To quantify individual differences in learning among mice, a variant of the procedures previously reported was used (Matzel et al. 2003). The animals were tested in a series of five independent learning tasks (Lashely III maze, passive avoidance, spatial water maze, odor discrimination, and fear conditioning) that place unique sensory, motor, motivational, and information processing demands on the animals.

\section{Lashley III maze}

This maze consists of a start box, three interconnected alleys and a goal box. Previous studies have shown that over successive trials, the latency to find the goal box decreased, as well as the number of wrong turns. When extramaze cues are minimized, the animals tend to use egocentric methods to locate the goal box (e.g., fixed motor patterns).

A Lashely III maze scaled down for use with mice was constructed from black Plexiglas and located in a dimly lit room (10 Lux at the floor of the maze). A 3-cm-diameter, white circle was located in the center of the goal box, and $45 \mathrm{mg}$ Bioserve food pellets (rodent grain) was placed in the cup to motivate the animal's behavior.

Food-deprived animals were acclimated and trained on two successive days. Prior to acclimation, they were exposed to three pellets of the reinforcer in their home cage. On the acclimation day, each mouse was confined in each of the first three alleys of the maze for $4 \mathrm{~min}$ and the final alley containing the goal box for $6 \mathrm{~min}$-where three pellets were placed in the goal box. At the end of each period, the animal was allowed to walk into the next alley before being confined. On the training day, the animals were placed in the start box and allowed to freely navigate the maze, during which time their latency to locate their food and the number of wrong turns were recorded. Upon successfully consuming the pellet, the animal was returned to its home cage for a 25-min intertrial interval during which time the maze was cleaned. The animals completed five trials during training. For purpose of ranking the mice, the number of trials until they made no more then a total of eight errors on two successive trials served as a learning index.

\section{Passive avoidance}

In this assay, animals learn to suppress their exploratory tendency in order to avoid aversive stimuli. The animals are placed on a platform, and if they step down, they are administered aversive stimuli, in this case a bright light, noise, and vibration.

A chamber with a white grid floor $16 \times 12 \mathrm{~cm}$ (length $\times$ width) and that is illuminated by a dim red light was used for both acclimation and testing. An enclosed platform $(70 \times 45 \times 45 \mathrm{~cm}$, length $\times$ width $\times$ height $)$ constructed of black Plexiglas and elevated $5 \mathrm{~cm}$ above the grid floor was located at the back of the chamber. It has only one opening facing the grid floor, which allows the animal to step down onto the floor. The exit from the platform can be blocked remotely by a clear Plexiglas guillotine-style door. When an animal leaves the platform and makes contact with the grid floor, it initiates the aversive stimuli.

Animals are placed on the platform with the door closed, confining them in the enclosure. After $5 \mathrm{~min}$, the door is opened, and the latency of the animal to leave the platform and make contact with the floor was recorded. After they make contact, the aversive stimuli are initiated and the platform is lowered, exposing them to the stimuli for $4 \mathrm{sec}$ after which they are allowed access to the enclosure again. They were then again confined on the platform for $5 \mathrm{~min}$, after which the door opened and their latency to walk onto the grid floor was recorded for a second time. For purpose of ranking the animals, the ratio of the poststep-down latency to pre-step-down latency served as the index of learning.

\section{Spatial water maze}

This task requires the animals to locate a submerged platform in a pool of opaque water using only spatial cues. The animals are motivated by their aversion to water. The latency and the path length to locate the platform decrease over successive trials, despite entering the pool from different locations.

A round pool (140 cm diameter, $56 \mathrm{~cm}$ deep) was filled to within $20 \mathrm{~cm}$ of the top with water that is clouded with a nontoxic, water-soluble black paint. A hidden 14 -cm-diameter, black platform was located in a fixed position $1 \mathrm{~cm}$ below the surface of the water. The pool was enclosed by a ceiling-high black curtain on which three different light patterns were fixed at various positions.

On the day prior to training, each animal was confined to the platform by a clear Plexiglas cylinder that fits around the platform for $360 \mathrm{sec}$. On the next two training days, the animals were started from one of three positions for each trial such that no two subsequent trials start from the same position. The animal is said to have successfully located the platform when it remains on the platform for $10 \mathrm{sec}$. After locating the platform or swimming for $90 \mathrm{sec}$, the animals were left or placed on the platform for $10 \mathrm{sec}$. After which, they were removed for $10 \mathrm{~min}$ and placed in a holding box before the start of the next trial. Each animal completed 10 total trials (six on the first training day, four on the second). The latency to find the platform was recorded for each trial. For purpose of ranking, the average of the third and fourth trial was used.

\section{Associative fear conditioning}

Animals receive a tone (CS) paired with a mild foot shock (US). To measure the conditioned fear responses, those are elicited by the CS through lick suppression.

Two distinct experimental chambers were used to avoid interactions between the training context and the CS at the time of testing. Each box $(32 \times 32 \times 28 \mathrm{~cm}$; length $\times$ width $\times$ height $)$ was contained within a sound- and light-attenuating chamber. The training box was brightly lit with clear Plexiglas walls and no lick tube and had a stainless steel grid floor (5-mm spacing). The test chamber on the other hand was dimly lit, the walls were covered with an opaque pattern of alternating black and white vertical stripes, and the floor was formed in a grid of stainless $1.5-\mathrm{m}$ rods arranged in $8-\mathrm{mm}$ squares. A water lick tube that protruded through a small hole in the wall was present. In the 
training chamber, a 0.6-mA, constant-current footshock could be delivered through the floor.

Water-deprived animals were acclimated to the training and testing contexts by placing each animal in both boxes for $30 \mathrm{~min}$ on the day before training. Training on the subsequent day occurred in a single 40-min session, during which the animals received two tone-shock pairings after 15 min and after $30 \mathrm{~min}$. They were then returned to their home cages for $60 \mathrm{~min}$, after which they were reacclimated to the test chamber for $20 \mathrm{~min}$. The following day, during testing the animals were placed in the testing chamber, and after completion of 25 licks, the CS was presented and stayed on until the animal completed an additional 25 licks or times out at $600 \mathrm{sec}$.

\section{Odor discrimination}

Rodents are adept at using odor to guide their reinforced behavior. This task is modified from one used by Sara et al. (2001) but scaled down for use with mice. In this task, mice navigate through a field using unique odors to guide them. The animals learn to choose the food cup that contains the target smell when given three choices. The food cup locations are rotated, but the accessible food is always marked by the target odor (in this case mint).

The odor discrimination chamber consisted of a black Plexiglas 60-cm-square field with $30-\mathrm{cm}$-high walls, which was located in a dimly lit room with good ventilation. Three aluminum food cups were placed in three corners. Only the target cup had the food accessible. The other two cups had food located in a covered hole drilled into the side with a ventilation hole, allowing the mice to smell the food but not access it. One 30-mg portion of chocolate-flavored puffed rice acted as a reinforcer and was placed in a depression on top of the target cup. A cotton-tipped laboratory swab, which was loaded before each trial with $25 \mu \mathrm{L}$ of lemon-, mint-, or almond-flavored extract extended vertically from the back corner of each cup. Mint was always the target odor.

Each animal had $1 \mathrm{~d}$ of acclimation and $1 \mathrm{~d}$ of testing. The night prior to the acclimation day, food was removed from each animal. The next day each mouse was placed in the box for 20 min without the cups. At the end of the day, each animal received three pieces of the reinforcer. On the training day, each animal received four trials in which they were placed in the corner without a food cup. On the first trial, an additional reinforcer was placed on the edge of the cup; other than that, all of the trials are identical. At the end of each trial, the food cups were rearranged, but mint always remained as the target odor. For each trial, both latency to locate the food and number of errors were recorded (where an error is making contact with or smelling an incorrect food cup).

\section{Visual discrimination}

In this task, the animals learned to choose the target symbol among three possible choices to locate food (Fig. 4). The procedure proceeded exactly as specified for odor discrimination. However, the visual discrimination box was made to be distinct from the odor context by the addition of bright white stripes on the walls. The total number of initial training trials was also increased from four to eight trials since mice tend to learn about odor cues more quickly then visual cues.

\section{Cues Used in the Visual Discrimination Task}
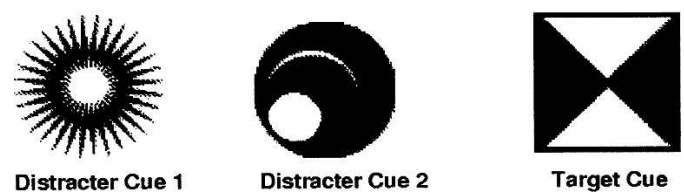

Figure 4. Cues used in visual discrimination task. The square-shaped cue was designated the target cue and was associated with food across trials.

\section{Assessing selective attention}

\section{Complex discrimination}

To assess differences in selective attention, it was first necessary to train the animals to perform odor discrimination and visual discrimination in two related yet distinct contexts (context 1 and context 2 respectively) - see Behavioral Training and Testing. Following initial training (for the assessment of learning rates) the animals were given additional overtraining trials until they reached an asymptotic level of performance (defined as a total of two errors or less over three trials). Following these overtraining trials, the animals performed a complex discrimination task in which the odor cues from context 1 were introduced into context 2 - acting as distracters. The animals also performed the opposite discrimination task in which the visual cues from context 2 acted as distracters in locating the odor target in context 1 . Total numbers of errors were once again recorded. Any observed performance deficit immediately following the introduction of these salient distracter cues was noted. It was expected that if the ability to selectively attend to task relevant cues and ignore distracters is related to general learning, then the errors made during this manipulation should correlate with the animals' average rank in the learning battery.

\section{Assessing short-term memory duration}

In order to assess short-term memory duration in the absence of a significant memory load, it was first necessary to train the animals in the reinforced alternation task. Acquisition in this task, however, was not included in the learning battery since it was observed that some animals had a tendency to spontaneously alternate (and hence did not require extensive learning) while others did not.

\section{Reinforced alternation}

In this task, the animals were required to choose between entering one of two arms that intersected to form the top of a " $\mathrm{T}$ ". A food reinforcer was present in the end of one arm. The location of the reinforcer shifted to the alternate arm after each successful retrieval of food. In order to perform efficiently in such a task, the animals had to alternate choices on successive trials (win-shift) in order to minimize the amount of effort it required to locate the food.

\section{Apparatus}

The apparatus consisted of a start arm $(7.5 \mathrm{~cm}$ wide $\times 17 \mathrm{~cm}$ long) that intersected at the middle of an alley that forms the top of a "T" (92 cm long $\times 6 \mathrm{~cm}$ wide). The entire maze was enclosed in a 5-cm-high wall. The initial segment of the start arm was segregated by a guillotine door that is remotely operated by the experimenter. This segment of the arm constituted the start box. At the entry of each choice arm, there was another experimenteroperated door. On the wall of the right arm, there were vertical white strips an inch apart. On the wall of the left arm, there were vertical strips. These strips will be used to help the animal discriminate between the arms.

\section{Training}

Training was conducted over three consecutive days. On day 1, animals were acclimated to the maze and allowed to make four forced choices. On the first exposure, the animal was held in the start box for $30 \mathrm{sec}$, after which it was allowed to traverse the maze; the door into the left arm was locked closed, and the right door was open. An 18-mg Noyes rodent pellet was located in the food cup in the right arm. After consuming the food, the animal was returned to the start box for a 20-sec intertrial interval (ITI). On the second exposure, this procedure was repeated, but the right door was locked and the left door open. After a 20-sec ITI, this sequence was repeated for two additional exposures. Through this sequence of four forced choices, the animals were acclimated to the maze.

On the subsequent days, animals were trained. On all training trials, each choice door was fully open. On trial 1 , a reinforcer 
was available in both food cups, and the animal could make a free choice. On the second trial, reinforcement was available in the arm not entered on the first trial. If an animal chose the correct arm, the location of the reinforcer alternated on the following trial. If an incorrect choice was made, the animal was allowed to correct its mistake and locate the food in the other arm. In either case, after the reinforcer was consumed, the animal was placed back in the start box to begin a 20-sec ITI. Animals' choices were recorded on each trial for 12 trials. Day 3 proceeded exactly like day 2 for 12 additional trials.

\section{Testing}

After all the animals were reliably performing this task, the shortterm delay manipulation was introduced. During this manipulation, the ITI was increased by 20 sec following an animal's correct choice and was decreased by $20 \mathrm{sec}$ following an incorrect choice. This procedure of "one up, one down" continued for 15 trials, at which point the delay at which the animal had stabilized was recorded (operationalized as the average delay across the last four trials).

\section{Assessing short-term memory capacity}

In order to assess short-term memory capacity in the absence of confounds such as spatial ability, the animals were trained to asymptote in an explicitly nonspatial radial arm maze.

\section{Nonspatial radial arm maze}

In this maze, there were visual cues attached to food cups located at the end of six radial arms. After an animal located a reinforcer, the food cup/visual cues were randomly relocated to new arms while the animal was confined in the center of the maze. Therefore, in order to efficiently navigate the maze (not return to an arm already visited), the animals must remember which visual symbols they had already visited. Due to the random nature of the shuffling of the cues after each choice, spatial cues could not provide "useful" information.

\section{Apparatus}

The apparatus consisted of a central hub (12-cm diameter) with eight arms radiating out from it $(10 \mathrm{~cm}$ long). The animal could be confined in the central hub by an experimenter-operated door. Food cups with a $1.5-\mathrm{cm}$-high lip and containing a single 45-mg Noyce pellet were located at the end of six on the eight arms. Attached to each cup was a semitransparent, distinct visual cue. At the ends of the remaining two arms were transparent, Plexiglas walls. A florescent tubular light was located beneath the maze and illuminated the visual cues. This design allowed the light to enter the maze through the visual cues (making them the most salient visual objects in the maze).

\section{Training}

On the first day of training, the animals were acclimated to the maze. During this period they were placed in the center of the maze with the doors closed and all the arms baited with 45-mg Noyse rodent pellets. After $10 \mathrm{sec}$ the doors were opened, and the animal was allowed to traverse the maze freely. Once the animal had eaten all of the pellets, it was removed. On day 2 the animals were started in the center of the maze with the doors closed and all the arms baited. After each pellet the animal consumed, the animal was confined to the center of the maze for $30 \mathrm{sec}$. During this period, the lights went off and the cues were semi-randomly sorted. This continued until it had located and eaten all of the pellets. An error was counted as any entrance into an arm that did not contain food. The animals were run for one trial a day over an $11 \mathrm{~d}$ period. Following this training, the number of errors made by the animals reached an asymptotic level. Performance at this point was taken to reflect in part the limits of their shortterm memory capacity.

\section{Acknowledgments}

We thank Drs. Arnold Glass and Timothy Otto for their comments on an earlier version of this manuscript. This work was supported by a grant from the National Institute of Aging (AG022698-01) to L.D.M.

\section{References}

Ackerman, P.L. 1987. Individual differences in skill learning: an integration of psychometric and information processing perspectives. Psychol. Bull. 102: 3-27.

Ackerman, P.L. 2005. Ability determinants of individual differences in skilled performance. In Cognition and intelligence: Identifying the mechanisms of the mind (eds. R.J. Sternberg and J.E. Pretz), pp. 142-159. Cambridge University Press, Cambridge, UK.

Baddeley, A.D. 1986. Working memory. Oxford University Press, Oxford, UK.

Baddeley, A.D. 2003. Working memory looking back and looking forward. Nat. Rev. Neurosci. 4: 829-839.

Blinkhorn, S. 2003. Neuroscience: Of mice and mentality. Nature 24: 1004-1005.

Colom, R., Rbello, I., Palacios, A., Juan-Espinosa, M., and Kyllonen, P.C. 2004. Working memory is (almost) perfectly predicted by $\mathrm{g}$. Intelligence 32: 277-296.

Conway, A.R. and Engle, R.W. 1995. Working memory and retrieval: A resource-dependent inhibition model. J. Exp. Psychol. Gen. 123: 354-373.

Conway, A.R., Cowan, N., Bunting, M., Therriault, D.J., and Scott, R.B. 2002. A latent variable analysis of working memory capacity, short-term memory capacity, processing speed, and general fluid intelligence. Intelligence 30: 163-183.

Conway, A.R., Kane, M.J., and Engle, R.W. 2003. Working memory capacity and its relation to general intelligence. Trends Cogn. Sci. 7: 547-552.

Dayan, P., Kakade, S., and Montague, P.R. 2000. Learning and selective attention. Nat. Neurosci. 3: 1218-1223.

Engle, R.W., Tuholski, S.W., Laughlin, J.E., and Conway, A.R. 1999. Working memory, short-term memory, and general fluid intelligence: A latent variable approach. J. Exp. Psychol. Gen. 128: 309-331.

Galsworthy, M.J., Paya-Cano, J.L., Monleon, S., and Plomin, R. 2002. Evidence for general cognitive ability $(g)$ in heterogeneous stock mice and an analysis of potential confounds. Genes Brain Behav. 1: 88-95.

Gray, J.R., Chabris, C.F., and Braver, T.S. 2004. Neural mechanisms of general fluid intelligence. Nat. Neurosci. 6: 316-322.

Haier, R.J., Buchsbaum, M.S., Abel, L., Tang, C., and Siegel, B. 2004. Intelligence and changes in the regional cerebral glucose metabolic rate following learning. Intelligence 16: 415-426.

Jarrold, C. and Towse, J.N. 2006. Individual differences in working memory. Neuroscience 139: 39-50.

Kolata, S., Light, K., Townsend, D.A., Hale, G., Grossman, H.C., and Matzel, L.D. 2005. Variations in working memory capacity predict individual differences in general learning abilities among genetically diverse mice. Neurobiol. Learn. Mem. 84: 241-246.

Locurto, C. and Scanlon, C. 1998. Individual differences and a spatial learning factor in two strains of mice (Mus musculus). J. Comp. Psychol. 112: 344-352.

Locurto, C., Fortin, E., and Sullivan, R. 2003. The structure of individual differences in heterogeneous stock mice across problem types and motivational systems. Genes Brain Behav. 1: 40-55.

Mackintosh, N.J. 1998. IQ and human intelligence. Oxford University Press, Oxford, UK.

Matzel, L.D., Han, Y.R., Grossman, H., Karnik, M.S., Patel, D., Scott, N., Specht, S.M., and Gandhi, C.C. 2003. Individual differences in the expression of a "general" learning ability in mice. J. Neurosci. 23: 6423-6433.

Sara, S.J., Roullet, P., and Przybyslawski, J. 1999. Consolidation of memory for odor-reward association: $\beta$-Adrenergic receptor involvement in the late phase. Learn. Mem. 6: 88-96.

Süß, H.M., Oberauer, K., Wittmann, W.W., Wilhelm, O., and Schulze, R. 2002. Working memory capacity explains reasoning ability-and a bit more. Intelligence 30: 261-288.

Received August 16, 2006; accepted in revised form November 10, 2006. 


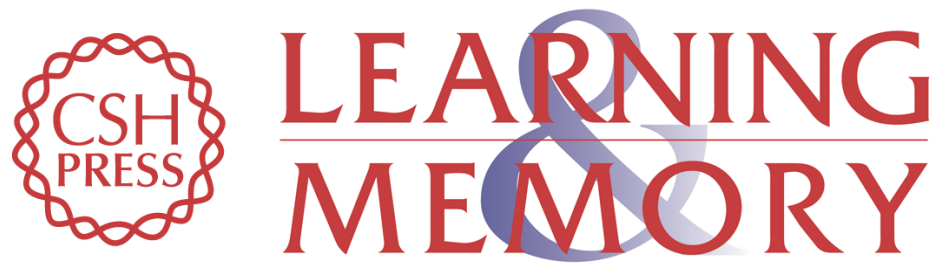

\section{Selective attention is a primary determinant of the relationship between working memory and general learning ability in outbred mice}

Stefan Kolata, Kenneth Light, Henya C. Grossman, et al.

Learn. Mem. 2007, 14:

Access the most recent version at doi:10.1101//m.408507

References This article cites 19 articles, 2 of which can be accessed free at:

http://learnmem.cshlp.org/content/14/1-2/22.full.html\#ref-list-1

License

Email Alerting Receive free email alerts when new articles cite this article - sign up in the box at the Service top right corner of the article or click here. 